\title{
CTBP1 depletion on prostate tumors deregulates miRNA/mRNA expression and impairs cancer progression in metabolic syndrome mice
}

\author{
Guillermo Nicolás Dalton ${ }^{1}$, Cintia Massillo ${ }^{1}$, Georgina Daniela Scalise ${ }^{1}$, Rocío Duca', Juliana Porretti ${ }^{1}$, Paula Lucia Farré , \\ Kevin Gardner ${ }^{2}$, Alejandra Paez ${ }^{3}$, Geraldine Gueron $\mathbb{0}^{3}$, Paola De Luca ${ }^{1}$ and Adriana De Siervi ${ }^{1}$
}

\begin{abstract}
About 20\% of prostate cancer (PCa) patients progress to metastatic disease. Metabolic syndrome (MeS) is a pathophysiological disorder that increases PCa risk and aggressiveness. C-terminal binding protein (CTBP1) is a transcriptional corepressor that is activated by high-fat diet (HFD). Previously, our group established a MeS/PCa mice model that identified CTBP1 as a novel link associating both diseases. Here, we integrated in vitro (prostate tumor cell lines) and in vivo (MeS/PCa NSG mice) models with molecular and cell biology techniques to investigate MeS/CTBP1 impact over PCa progression, particularly over cell adhesion, mRNA/miRNA expression and PCa spontaneous metastasis development. We found that CTBP1/MeS regulated expression of genes relevant to cell adhesion and PCa progression, such as cadherins, integrins, connexins, and miRNAs in PC3 xenografts. CTBP1 diminished PCa cell adhesion, membrane attachment to substrate and increased filopodia number by modulating gene expression to favor a mesenchymal phenotype. NSG mice fed with HFD and inoculated with CTBP1-depleted PC3 cells, showed a decreased number and size of lung metastases compared to control. Finally, CTBP1 and HFD reduce hsa-mir-30b-5p plasma levels in mice. This study uncovers for the first time the role of CTBP1/MeS in PCa progression and its molecular targets.
\end{abstract}

\section{Introduction}

Prostate cancer (PCa) is the second most diagnosed cancer type and the fifth cause of death by cancer among males worldwide ${ }^{1}$. Most PCa-related deaths are due to advanced disease, which results from any combination of lymphatic, hematogenous, or contiguous local spread ${ }^{2}$. About $90 \%$ of patients in the final stages of $\mathrm{PCa}$, known as castration resistant prostate cancer (CRPC), will develop

\footnotetext{
Correspondence: Adriana De Siervi (adesiervi@dna.uba.ar)

'Laboratorio de Oncología Molecular y Nuevos Blancos Terapéuticos, Instituto

de Biología y Medicina Experimental (IBYME), CONICET, Argentina

${ }^{2}$ Department of Pathology and Cell Biology, Columbia University Medical

Center, 630 W. 168th Street, New York, NY 10032, USA

Full list of author information is available at the end of the article.

Edited by C. Munoz-Pinedo
}

bone metastases which dramatically reduce patient survival and quality of life ${ }^{3}$.

Metabolic syndrome (MeS) is one of the most widely prevailing health concerns worldwide. It is a cluster of pathophysiological disorders whose diagnose requires the detection of, at least, three of the following factors: visceral adiposity, high triglycerides, low-high density lipoprotein (HDL) cholesterol levels, high-blood pressure, and elevated fasting glucose levels ${ }^{4}$. Latest estimates indicate a worldwide prevalence ranging between 10 and $40 \%$, depending on lifestyle and genetic background ${ }^{5}$. Diet, lifestyle, and genetic background not only affect MeS, there is an increasing body of evidence showing that these factors play a crucial role in PCa risk and progression ${ }^{6-8}$. Likewise a recent meta-analysis found a significant 
correlation associating $\mathrm{MeS}$ with more aggressive $\mathrm{PCa}$ tumors and biochemical recurrence ${ }^{9}$. Nonetheless, the molecular players responsible for the effect of $\mathrm{MeS}$ on the progression/aggressiveness of $\mathrm{PCa}$ tumors are yet to be completely identified.

Recent years have seen an overflow of reports regarding miRNAs role in cancer. Many reviews have been published on miRNAs deregulation in cancer, both as cause and consequence, and as possible biomarkers or therapeutic molecules ${ }^{10-13}$.

Previously our group identified C-terminal binding protein 1 (CTBP1) as a link between $\mathrm{MeS}$ and $\mathrm{PCa}^{14,15}$. CTBP1 is a transcriptional corepressor of many tumor suppressor genes. Binding either NAD + or NADH is necessary for CTBP1 activation; however, CTBP1 affinity is 100-fold higher for NADH making it a molecular sensor of the metabolic state of the cell ${ }^{16}$. We previously generated a murine model of MeS and PCa by chronically feeding animals with high-fat diet (HFD). This model allowed us to identify novel pathways regulated by CTBP1 on a MeS environment ${ }^{14}$. CTBP1 depletion in prostate xenografts developed in MeS $n u / n u$ mice dramatically decreased tumor growth and modulated cell adhesion, metabolic process, and cell cycle-related genes ${ }^{14}$. Moreover, we recently described a novel regulation of cell adhesion and epithelial-to-mesenchymal transition (EMT) in PCa cells by the repression of chloride channel accessory 2 (CLCA2) mediated by CTBP1 and miR-196b-5p. Also, we demonstrated that CLCA2 is a target of miR196b-5 $\mathrm{p}^{15}$.

In this work our aim was to understand CTBP1 and related miRNAs role on $\mathrm{PCa}$ progression. We demonstrated that CTBP1 decreases the in vitro adhesive capabilities of a panel of $\mathrm{PCa}$ cell lines through the modulation of genes like Cadherin 1 (CDH1), Integrin Subunit Beta 4 (ITGB4), and Vimentin (VIM) among others. Consistently, CTBP1 favors a mesenchymal and pro-invasive phenotype. Using a $\mathrm{MeS}$ and spontaneous PC3 metastasis in vivo model, we found that CTBP1 depletion on MeS mice impairs the development of lung metastases. In addition, we show that CTBP1 regulates a cluster of miRNAs that target cell adhesion genes, which could in turn impact over cell adhesion itself and ultimately on the onset of metastatic disease.

\section{Results}

\section{CTBP1 regulates expression of mRNAs and miRNAs} involved in cell adhesion on a PC3 and MeS in vivo model

We previously reported a mice model of $\mathrm{PCa}$ and $\mathrm{MeS}^{14}$. Briefly, male $n u / n u$ mice fed with control diet (CD) or HFD during 12 weeks, were s.c. inoculated with CTBP1 depleted (PC3.shCTBP1) or control (PC3.PGIPZ) PC3 cells. RNA isolated from xenografts grown on MeS mice was used to hybridize a whole-genome expression microarray (Affymetrix). Gene set enrichment analysis (GSEA) indicated that CTBP1 regulates many pathways ${ }^{14}$, being "cell adhesion" one of the more robustly represented. We selected a list from this set that included cancer progression related genes and validated it by realtime quantitative polymerase chain reaction (RT-qPCR). As shown on Fig. 1a, CTBP1 depletion and/or MeS increased the expression of cadherins ( $C D H 1$ and $C D H 3)$, integrins (ITGB4, ITGB6, and ITGA1), collagen type XVII alpha 1 (COL17A1), connexin (GJB5), serine protease (PRSS2), transglutaminase (TGM2), and lipocalin (LCN2).

We previously reported that CTBP1 regulates miRNAs ${ }^{15,17,18}$. Concisely, RNA samples obtained from the xenografts tumors described above were used to hybridize a miRNA expression microarray (Affymetrix) ${ }^{15}$. In this work, miRNA and mRNA microarray results were cross referenced. We used ChemiRs database to obtain lists of genes regulated by miRNAs on the array and compared them to the cell adhesion cluster from the mRNA array (Fig. 1b). miRNA expression was determined by miRNA RT-qPCR from CTBP1-depleted xenografts or control, grown in $\mathrm{CD}$ or HFD-fed mice (Fig. 1c). Interestingly, CTBP1 depletion downregulated miRNAs related to cell adhesion, such as hsa-miR-19b-3p and hsa-miR-454-3p. Other miRNAs, hsa-miR-30b-5p, hsa-miR-301a-3p, and hsa-miR-29c-3p were downregulated by MeS and, hsamiR-184, hsa-miR-140-5p, hsa-miR-335-5p, and hsa-miR6799-3p could not be validated by this method (Fig. 1c). Moreover, hsa-miR-205-5p, a known tumor suppressor miRNA, was highly upregulated in CTBP1-depleted tumors (Fig. 1c).

\section{CTBP1 diminishes cell adhesion by modulating gene expression}

To explore the effect of CTBP1 on cell adhesion, we transfected PC3, 22Rv1, LNCaP, C4-2, and DU 145 cells with control (PC3.pcDNA3) or CTBP1 (PC3.CTBP1) expression plasmids, and assessed cell adhesion to collagen coated- or noncoated plastic. As shown in Fig. 2a, b, CTBP1 overexpression significantly diminished cell adhesion in all PCa cell lines under study.

It was previously reported that CTBP1 interacts with ZEB1 transcription factor to repress the expression of genes involved in EMT process, such as $C D H 1^{19}$. Hence, we investigated CTBP1 and/or ZEB1 overexpression effect over cell adhesion. Although, CTBP1 and ZEB1 overexpression decreased cell adhesion, no synergy was observed between these two coregulators (Fig. 2c).

In order to understand the molecular mechanism for CTBP1 role in cell adhesion, we studied gene-expression modulation by CTBP1 in different adherent cell populations. PC3 cells were transiently transfected to overexpress CTBP1. Twenty-four hours later cells were harvested and seeded on culture plates for $60 \mathrm{~min}$. 
A
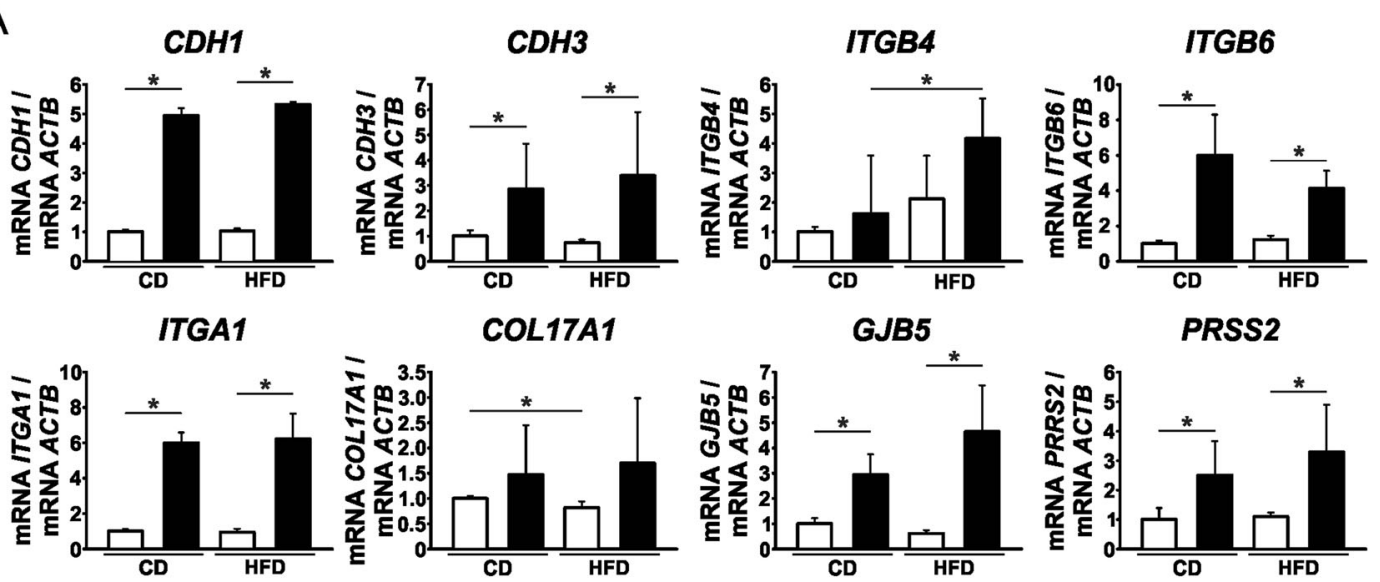

TGM2

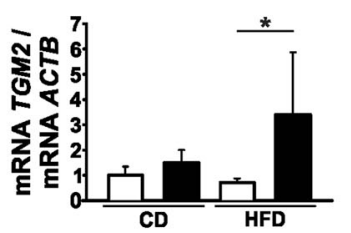

LCN2

CTBP1
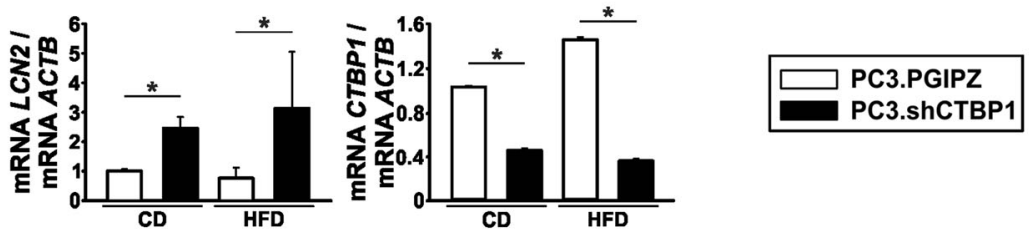

B

miRNA array Results

$\downarrow$

ChemiRs Analysis:

Genes regulated by miRNAs

"Cell adhesion" gene cluster from mRNA array $\downarrow$

miRNAs associated to the GO term Cell Adhesion

\begin{tabular}{|c|}
\hline hsa-miR-301a-3p \\
\hline hsa-miR-140-5p \\
\hline hsa-miR-454-3p \\
\hline hsa-miR-184 \\
\hline hsa-miR-6829-5p \\
\hline hsa-miR-6799-3p \\
\hline hsa-miR-335-5p \\
\hline hsa-miR-19b-3p \\
\hline hsa-miR-29c-3p \\
\hline hsa-miR-30b-5p \\
\hline hsa-miR-205-5p \\
\hline
\end{tabular}

C

hsa-mir-19b-3p

hsa-mir-454-3p

hsa-mir-30b-5p
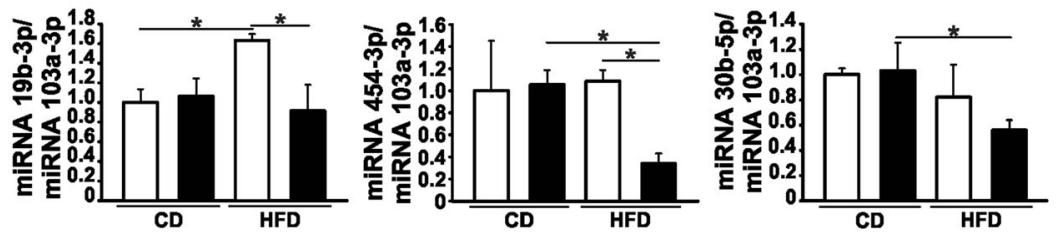

hsa-mir-301a-3p

hsa-mir-29c-3p

hsa-mir-184
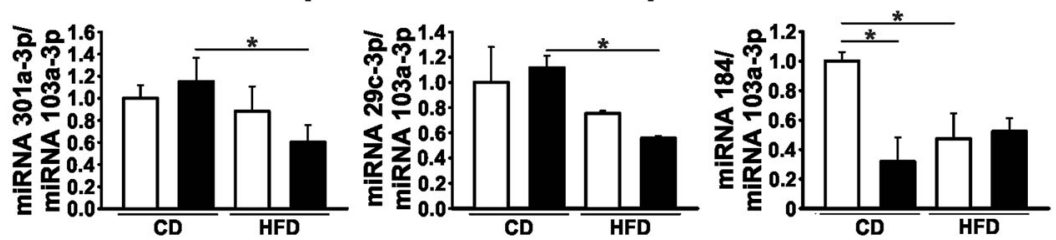

hsa-mir-140-5p

hsa-mir-335-5p
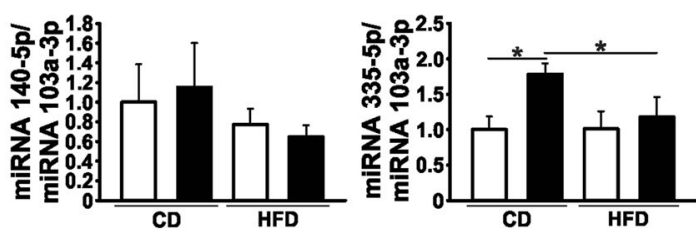

hsa-mir-6799-3p

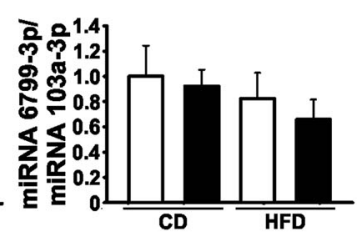

hsa-mir-205-5p
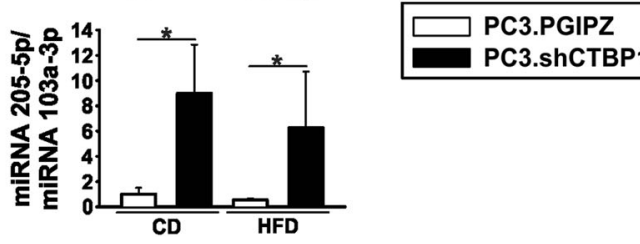

PC3.shCTBP1

Fig. 1 CTBP1 regulates mRNAs and miRNAs related to GO "cell adhesion" in xenografts grown on MeS mice. Expression of the indicated mRNAs (a) and miRNAs (c) in xenografts from CD or HFD nu/nu mice inoculated with PC3.shCTBP1 or PC3.PGIPZ cells were determined by RT-qPCR using specific primers. Data were normalized to ACTB and control for mRNAs or to hsa-miR-103a-3p and control for miRNAs. Bars represent the average and SD from three independent mice. Significance was analyzed by two-way ANOVA. ${ }^{*} p \leq 0.05$. b Flow chart and list of miRNAs associated to the GO term "cell adhesion" 

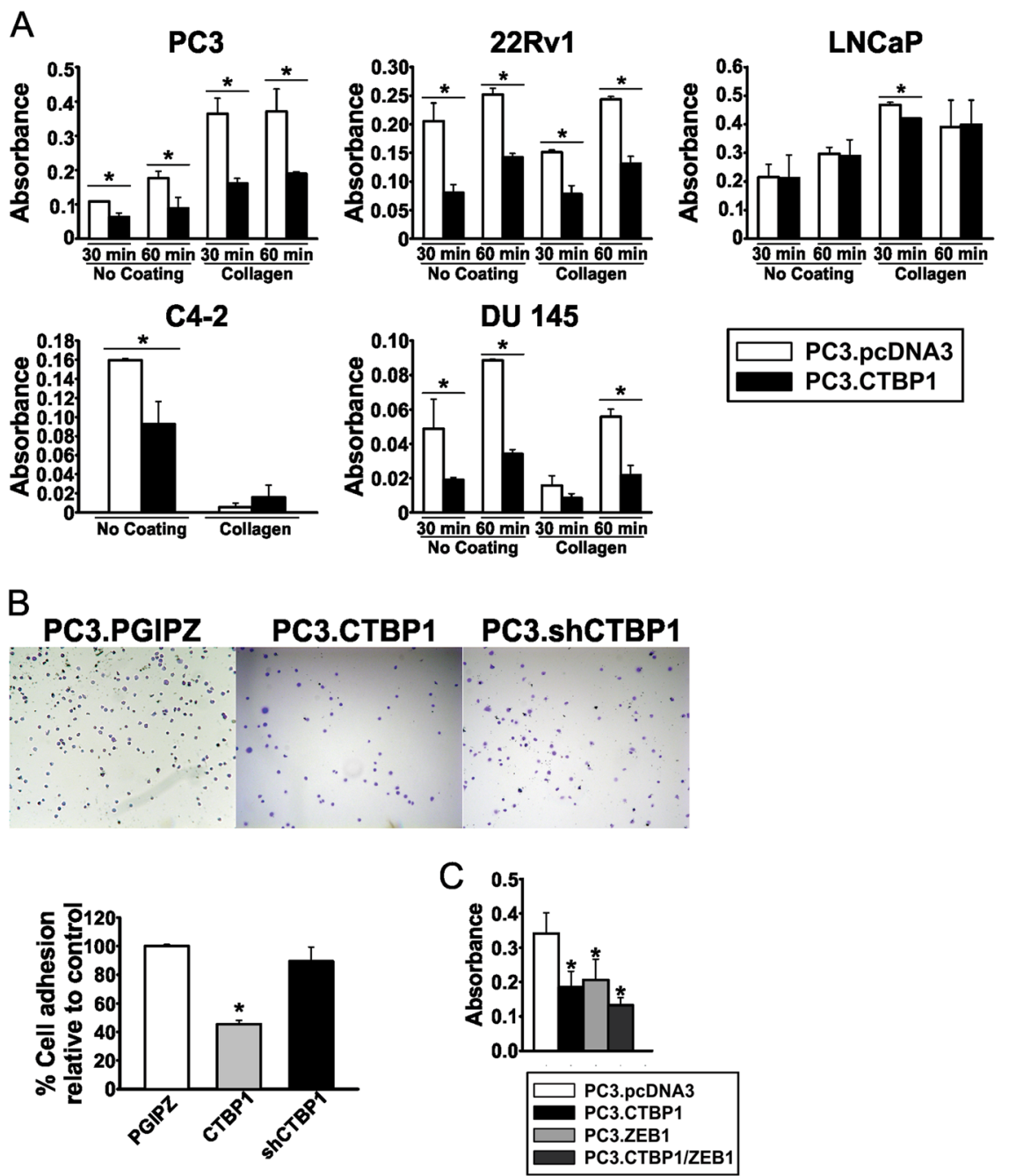

Fig. 2 CTBP1 diminishes cell adhesion in PCa cell lines. Cell adhesion assay. Different PCa cell lines, with varying levels of CTBP1 expression were tested. a PC3, 22RV1, LNCaP, C4-2, and DU 145 cell lines transfected with a CTBP1 expression (PC3.CTBP1) or control (PC3.PCDNA3) plasmids. C4-2 cell line was tested only for $60 \mathrm{~min}$. b PC3.shCTBP1 and its respective control PC3.PGIPZ. Top, a representative image of fixed cells stained with CV. Bottom, graphical representation of total amount of attached cells per treatment. c PC3 cells were transfected with a control, CTBP1 and/or ZEB1 expression plasmids. Adhesion was tested only for $60 \mathrm{~min}$. In all cases bars represent the average and SD of a representative experiment. Data were analyzed by one-way ANOVA. * $p \leq 0.05$

Culture medium was removed in order to eliminate the less adherent cell population and cells attached to the collagen matrix were harvested immediately or after $12 \mathrm{~h}$. RNA isolation followed by RT-qPCR analysis from adherent and total cell populations demonstrated that CTBP1 transcriptionally repressed CDH1, ITGB4, ITGB6, ITGA1, GJA5, GJB5, PRSS2, and TGM2 gene expression, while induced $C D H 3$ and VIM (Fig. 3a). Notably, CTBP1 overexpression favored a mesenchymal phenotype since $C D H 1$, an epithelial marker, was significantly repressed and VIM, a mesenchymal marker, was induced (Fig. 3a). These results are consistent with CTBP1 role modulating cell adhesion genes as was observed in the xenograft tumors.
Based on the strong repression of ITGB4 by CTBP1, we explored CTBP1 binding to its promoter. Chromatin immunoprecipitation (ChIP) assay demonstrated that CTBP1 bound to ITGB4 proximal promoter region (Fig. 3b).

\section{CTBP1 reduces cell membrane attachment to substrate and increases filopodia number}

To investigate the morphological alterations caused by CTBP1 regulation of cell adhesion, we explored the area of the cell membrane directly in contact with the substrate by rhodamine-phalloidin staining and confocal microscopy. No changes were found after CTBP1 overexpression; however, stable CTBP1 depletion lead to a 


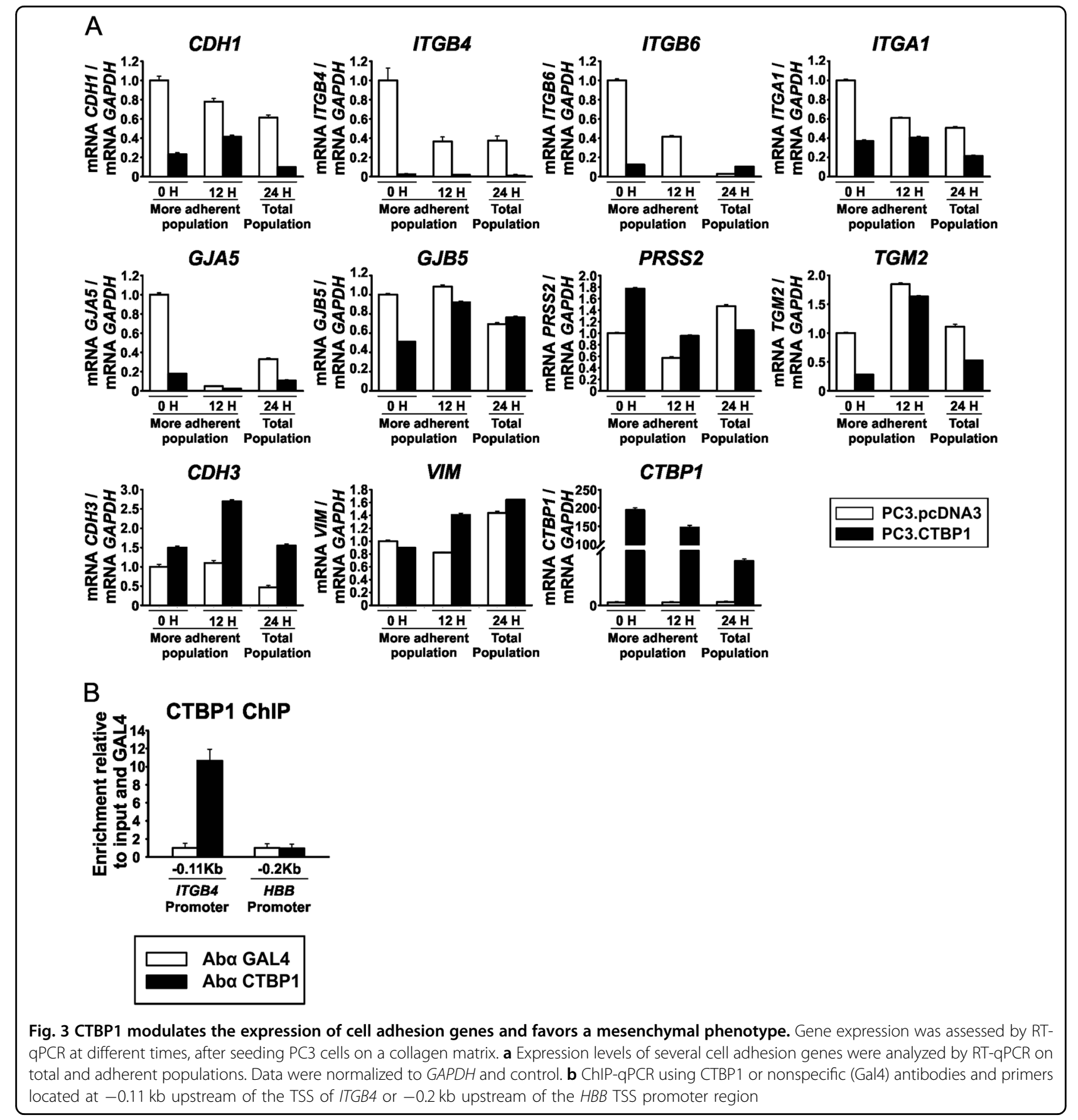

higher amount of cell membrane attached to the substrate, observed by the increase in cell length and width (Fig. 4). Filopodia are thin, finger-like and highly dynamic actin-rich membrane protrusions that extend out from the cell edge and correlate with metastatic potential. Interestingly, CTBP1 overexpression increased the number of cells with a high count of filopodia (Fig. 4b). These results demonstrated that CTBP1 plays a role in cell-cell and cell-ECM (extra cellular matrix) adhesion molecules loss, the shift to a mesenchymal phenotype and proinvasive cell morphology, suggesting the relevance of CTBP1 in the early stages of metastasis development.

CTBP1 depletion impairs PC3 metastasis in HFD/MeS-like mice

To further investigate CTBP1 role in metastasis, we generated an in vivo MeS and PC3 metastasis model. NOD Scid Gamma (NSG) mice were fed with HFD or CD. 


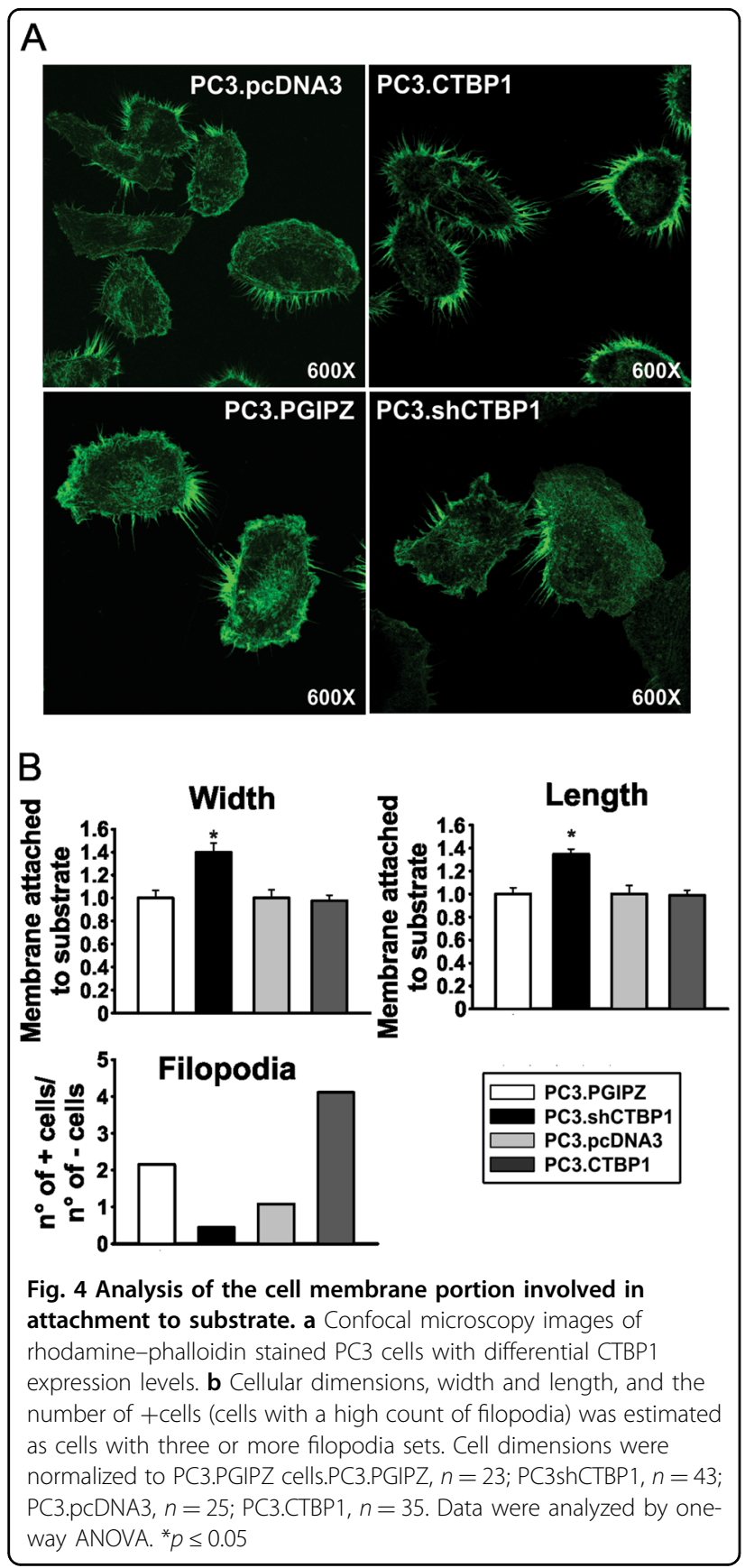

On the 12th week CTBP1-depleted or control PC3 cells were inoculated on the animal left flank. Four weeks later, mice were sacrificed and tumor and lung samples were excised for RNA isolation, followed by RT-qPCR, and histopathological analysis. Regarding tumor growth and body weight, no differences were observed between treatments (Fig. 5a). However, a dramatic body weight loss beginning about 15 days after cell inoculation was observed (Fig. 5a), suggesting metastatic disease in these mice.
CTBP1 depletion at the end of the experiment was confirmed by RT-qPCR (Fig. 5b). Although, CTBP2 expression levels were not affected by CTBP1 depletion in xenografts, HFD significantly increased CTBP2 mRNA levels compared to CD-fed mice group (Fig. 5b).

During the autopsy procedure, no macrometastases were observed in the mice visceral organs. However, we detected GAPDH gene expression by RT-qPCR using specific human primers in RNA samples from lungs, as a first evidence of micrometastasis. As shown on Fig. 5c, CTBP1 depletion on HFD-fed mice resulted in lowerGAPDH expression compared to other groups which indicated less human cells in lungs and, in turn, less metastasis. Histopathological analysis was also performed by hematoxylin and eosin (H\&E) staining confirming the metastasis reduction in CTBP1-depleted HFD-fed mice (Fig. 5d, Table 1). Furthermore, immunohistochemical (IHC) staining of CTBP1 showed that lung metastasis from CTBP1-depleted HFD mice had less CTBP1 protein compared to other mice groups (Fig. 5d).

Based on CTBP1 role in PCa cell adhesion and EMT, we also recovered circulating tumor cells (CTCs) from the peripheral blood of these mice. The colony formation capabilities of the CTCs were evaluated in a clonogenic assay. We found that CTBP1 depletion dramatically decreased the percentage of mice with CTC foci compared to PGIPZ control mice (Fig. 5e).

\section{PCa metastasis show high VIM and low-CDH1 and ITGB4 expression compared to primary tumors}

We compared the expression levels of key genes between primary tumors and human metastatic cells in the lung (Fig. 6). We found that metastatic cells had nearly null expression of the epithelial marker $C D H 1$ and the cell-ECM adhesion molecule ITGB4, expressed significantly higher levels of $V I M$ and CTBP2 and lower levels of CTBP1. However, PC3.shCTBP1 cells from lungs of HFD-fed mice, expressed similar levels of CTBP1 mRNA to those of the primary tumors (Fig. 6). Histological and RT-qPCR analysis of the primary tumors did not show differences between CD and HFD (data not shown).

\section{CTBP1 and HFD reduces hsa-mir-30b-5p plasma levels in mice}

We next tested the miRNA panel from Fig. 1b on mice plasma samples to detect circulating miRNAs in mice fed with CD or HFD and injected with CTBP1-depleted PC3 cells or control. After miRNA RT-qPCR, we only detected hsa-mir-30b-5p, hsa-mir-19b-3p, hsa-mir-205-5p and hsa-mir-140-5p (Fig. 6b). Interestingly, hsa-mir-30b was significantly decreased in plasma from HFD-fed mice (Fig. 6b). All the remaining miRNAs from Fig. 1b tested (hsa-miR-454-3p, hsa-miR-301a-3p, hsa-miR-29c-3p, hsamiR-184, hsa-miR-335-5p, and hsa-miR-6799-3p) were 
A

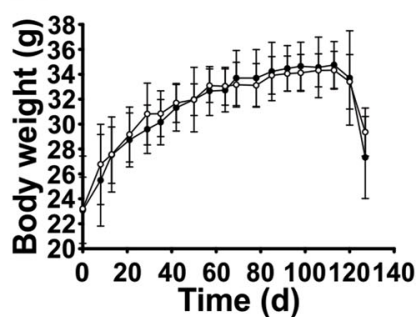

$\begin{array}{ccccc}0 & 20 \quad 40608010 \\ \text { Time (d) }\end{array}$

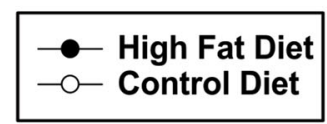

B

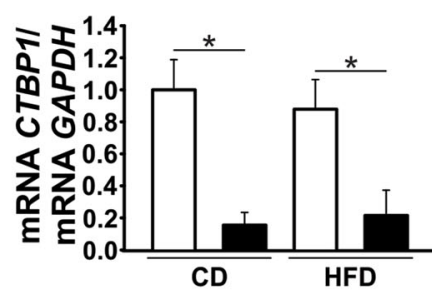

CTBP1
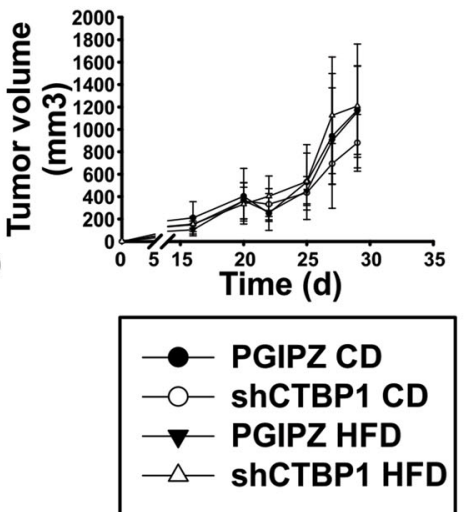

\section{CTBP2}
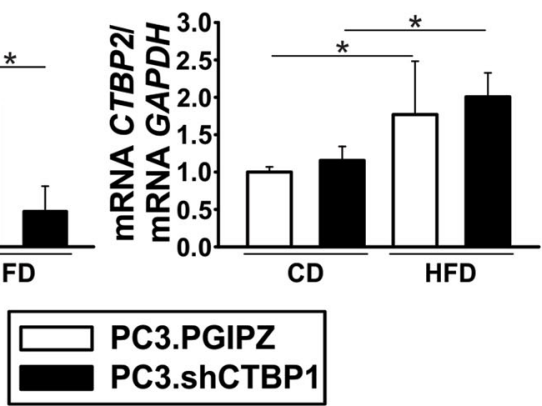

D

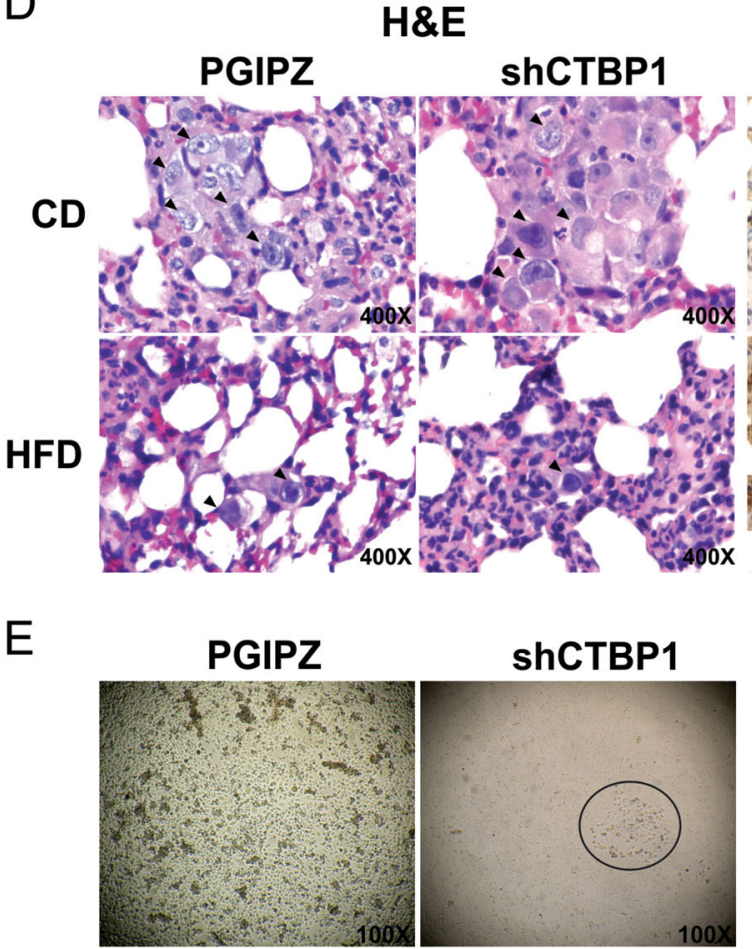

Fig. 5 (See legend on next page.)

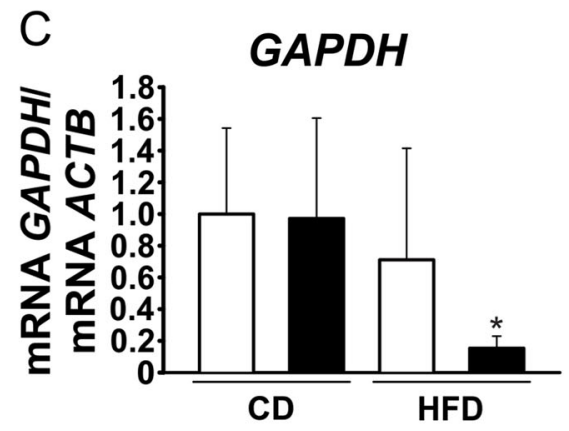

IHC CTBP1 PGIPZ ShCTBP1

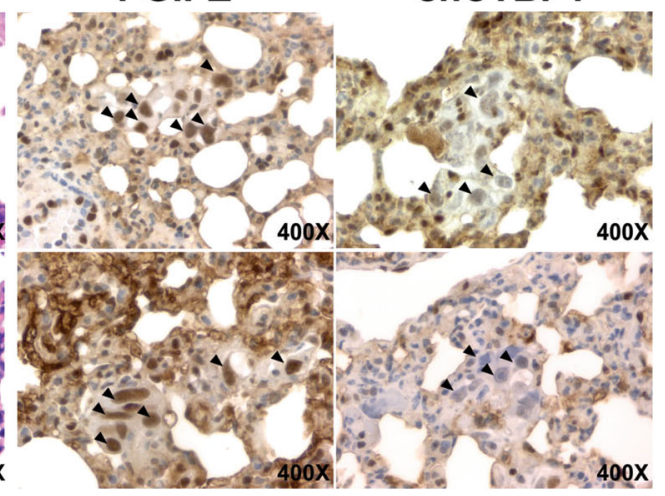


(see figure on previous page)

Fig. 5 PCa and MeS spontaneous metastasis model. Four-week-old NSG mice were fed a CD or HFD for the entirety of the experiment. a Body weight was measured once a week (left) and on the 12th week of diet mice were inoculated in the left flank with PC3.PGIPZ (control) or PC3.shCTBP1 (CTBP1 depleted) cells. Tumor size was measured three times a week (right). $\mathbf{b}$ CTBP1 and CTBP2 mRNA levels at the time of sample collection was assessed by RT-qPCR on tumor samples. $\mathbf{c}$ Lung metastasis burden was inferred by the mRNA levels of human GAPDH, detected in RNA samples from mice lung tissue. $\mathbf{d}$ H\&E (left) and CTBP1 IHC (inmunohistochemical) staining (right) from lungs of mice bearing xenografts, arrows indicate metastatic cells. e Representative images of CTCs harvested from PC3.PGIPZ $(n=1)$ or PC3.shCtBP1 $(n=3)$ inoculated NSG mice, 1 week after blood extraction. Bars represent average and SD of two independent experiments. Data were analyzed by two-way ANOVA. ${ }^{*} p \leq 0.05$

Table 1 Histological analysis of lung metastases

\begin{tabular}{llll}
\hline & & \multicolumn{2}{l}{ Lung metastases } \\
\cline { 3 - 4 } & & SCORE & CTBP1 \\
\hline CD & PGIPZ & 3.2 & Positive \\
& ShCtBP1 & 2.4 & Negative \\
& PGIPZ & 3 & Positive \\
& ShCtBP1 & 1 & Negative \\
\hline
\end{tabular}

NSG mice fed with HFD or CD were inoculated with PC3.PGIPZ or PC3. shCTBP1 cells. Lung metastasis score was determined based on metastatic focus number and size (cell number) by H\&E staining.

undetected in mice plasma. These results show for the first time that low-circulating miR-30b-5p correlates with high metastasis suggesting that this miRNA might have a role triggering metastasis in HFD-fed animals.

\section{Discussion}

The results presented on this work manifest the important role of CTBP1 and MeS on PCa progression and metastasis development. Metastasis is the major responsible of $\mathrm{PCa}$ mortality, and $\mathrm{MeS}$ has become a predominant risk factor for its incidence and progres$\operatorname{sion}^{2,9}$. In the present work, we presented new evidence linking both phenomena in CTBP1 and the plethora of genes it regulates either, directly or through miRNAs.

The role of CTBP1 in EMT and metastasis has been stated before in hepatocellular carcinoma ${ }^{20}$ and breast cancer ${ }^{18,21}$. More importantly, Wang et al. ${ }^{22}$ reported that mice injected through the tail vein with CTBP1-depleted PC3 cells had less metastasis development compared to control. Our results reinforced these findings using an in vivo model for cancer progression that recapitulates the metastatic process from the initial steps of EMT: cell detachment, invasion, and extravasation. Hence, we report for the first time that CTBP1 together with MeS play a crucial role inducing PCa progression from localized to metastatic disease.

Concerning EMT initiation, we showed that CTBP1 overexpression diminished $\mathrm{PCa}$ cell adhesion regardless of androgen sensitivity. This effect is most likely the result of CTBP1 repression of cell adhesion molecules, such as cadherins, connexins, and integrins. In the case of LNCaP cells, the effect of CTBP1 was subtle. Considering that these cells have higher-CTBP1 expression compared to the other PCa cell lines ${ }^{14}$, it might be that adhesion is at its lowest and could not be affected by CTBP1 overexpression.

In addition, we found that CTBP1 increases the number of filopodia favoring a pro-invasive phenotype. Accordingly, СТВP1 depletion lead to a higher proportion of cell surface attached to substrate and the derepression of cell adhesion genes in xenograft tumors.

Many of the genes regulated by CTBP1 and related to cell adhesion were novel targets with unknown role in PCa. Nonetheless we found some reports regarding their role in other types of cancer and diseases. As an example, COL17A1 is a transmembrane protein of the collagen family and a structural component of hemidesmosomes. It has been reported that COL17A1 promotes cell adhesion, which is consistent with our findings, but also increases cell motility and migration in wound healing process and in the leading front of some invasive carcinomas $^{23,24}$.

Considering these results, we explored CTBP1 effects on a mice model of MeS and spontaneous PCa metastasis. Remarkably, CTBP1 depletion impaired the development of lung metastases only on HDF fed mice.

Another important aspect is that CTBP2 expression was not altered as a consequence of CTBP1 depletion. This is not minor since CTBP1 and 2 share some overlapping functions and because one of the ways CTBP1 enters the nucleus is by forming a herterodimer with $\mathrm{CTBP} 2{ }^{25}$. However, CTBP1 and 2 might have different roles in metastasis progression, since $C T B P 2$ expression is higher in metastatic cells compared to localized tumors, while oppositely CTBP1 expression is low in metastasis. This might indicate that CTBP1 distinct functions from CTBP2 could be more relevant in the initial steps of metastasis, mainly loss of cellular adhesion and invasion increase, while CTBP2 would gain importance in the newly established metastatic niche.

In addition, we reported a miRNA cluster associated to cell adhesion regulated by CTBP1. These miRNAs are downregulated in CTBP1-depleted cells, which would be expected of miRNAs whose targets are upregulated in the 


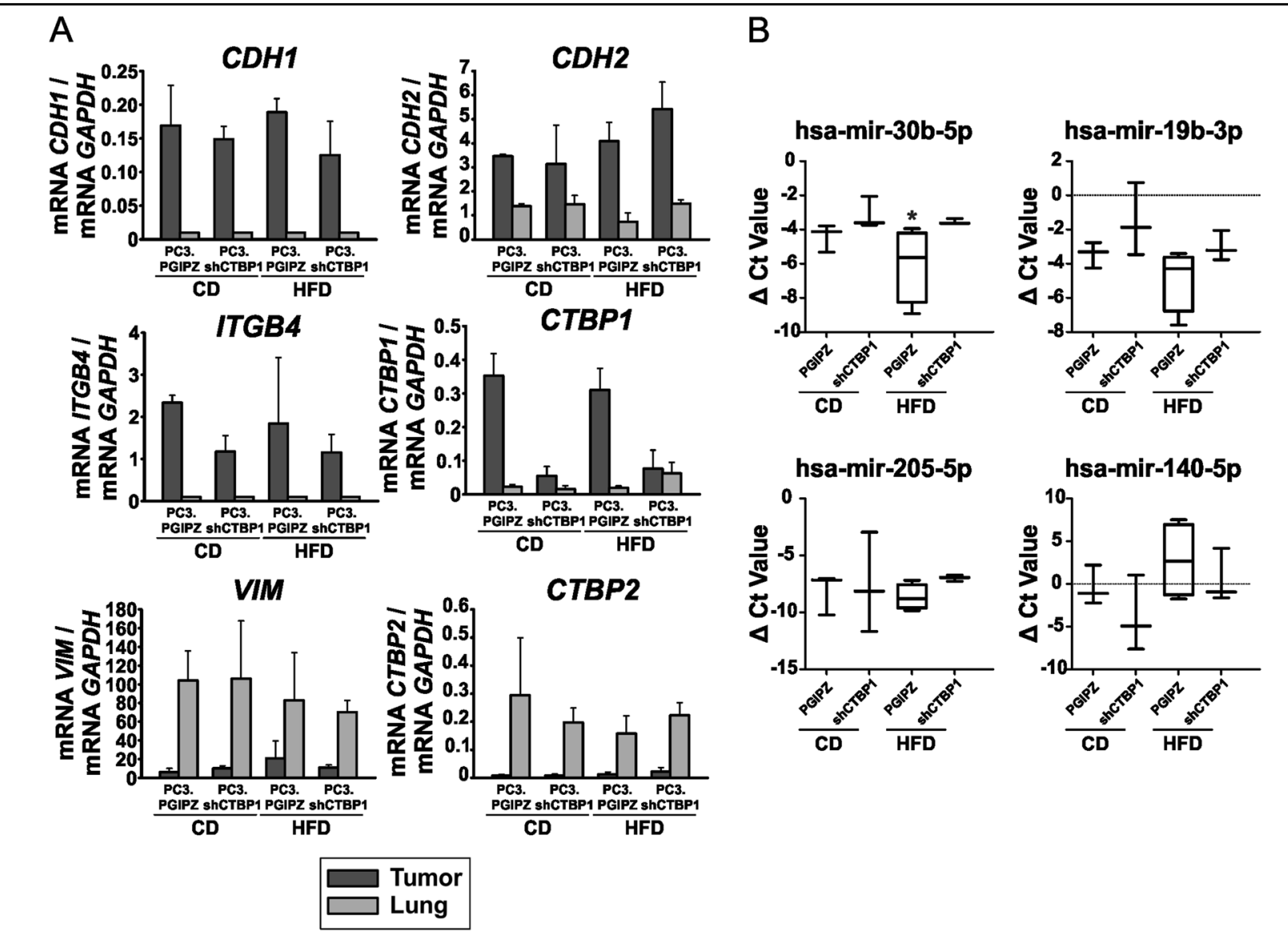

Fig. 6 Expression levels of relevant genes between localized tumor cells and metastatic cells. Circulating miRNAs in mice plasma. a Key genes involved in EMT-MET were analyzed by RT-qPCR between tumor and lung samples. CDH1 and ITGB4 expression levels were not detected on metastasis samples. Bars represent average and SD of a representative experiment. b Plasma samples were analyzed by RT-qPCR for the indicated miRNAs and normalized to spike in cel-39 synthetic miRNA ( $\Delta \mathrm{Ct}$ value $=\mathrm{Ct}_{\text {cel-39 }}-\mathrm{Ct}_{\text {sample }}$ ). Data were analyzed by $t$ test. ${ }^{*} p \leq 0.05$. Three mice were tested per experimental group

same conditions. In the future this miRNA cluster might be validated in patient samples with the ultimate goal of identifying potential biomarkers for $\mathrm{PCa}$ progression associated to MeS.

In particular, miR-205-5p was upregulated in CTBP1depleted cells. This miRNA shows much promise as a therapeutic tool since it has been reported as an antimetastatic miRNA for its role in EMT inhibition through ZEB1 silencing ${ }^{26}$. It is possible that an EMT regulatory loop which includes ZEB1, CTBP1, and miR-205-5p may exist. As cancer advances in a MeS context, CTBP1 expression and/or activity would increase, leading to the repression of miR-205-5p and the upregulation of ZEB1, kick-starting the EMT process.

The role of miRNAs in PCa has been reviewed previously by our group ${ }^{12}$. miRNAs are involved in different aspect of PCa progression from the acquisition of a CRPC phenotype, like the miR 221/222 cluster $^{27}$, to the promotion of EMT and the development of metastases as is the case of miR-22 role in repressing the epithelial marker $\mathrm{CDH} 1$ promoting cell invasion and migration ${ }^{28}$. Regarding the role of miR-30b-5p, the literature shows that this miRNA is cancer type dependent. Some authors show that miR-30b overexpression correlates with stage, metastatic potential, shorter time to recurrence and reduced overall survival on melanoma patients, and that ectopic overexpression of miR-30b/30d promotes a metastatic behavior in melanoma cells ${ }^{29}$. Other authors show that overexpression of miR-30b promotes apoptosis and suppresses tumor migration and invasion in gastric cancer cell lines ${ }^{30}$.

Regarding the genes regulated by CTBP1, it was not reported TGM2, COL17A1, GJB5, GJA5, ITGA1, and ITGB6 roles in PCa. However, oppositely to our findings, it was published that ITGB4 ${ }^{31,32}$ and $\mathrm{LCN} 2^{33,34}$ overexpression increased migration of $\mathrm{PCa}$ cell lines. Our findings characterize for the first time these genes function in vivo. Thus, the heterogeneity of cancer might 


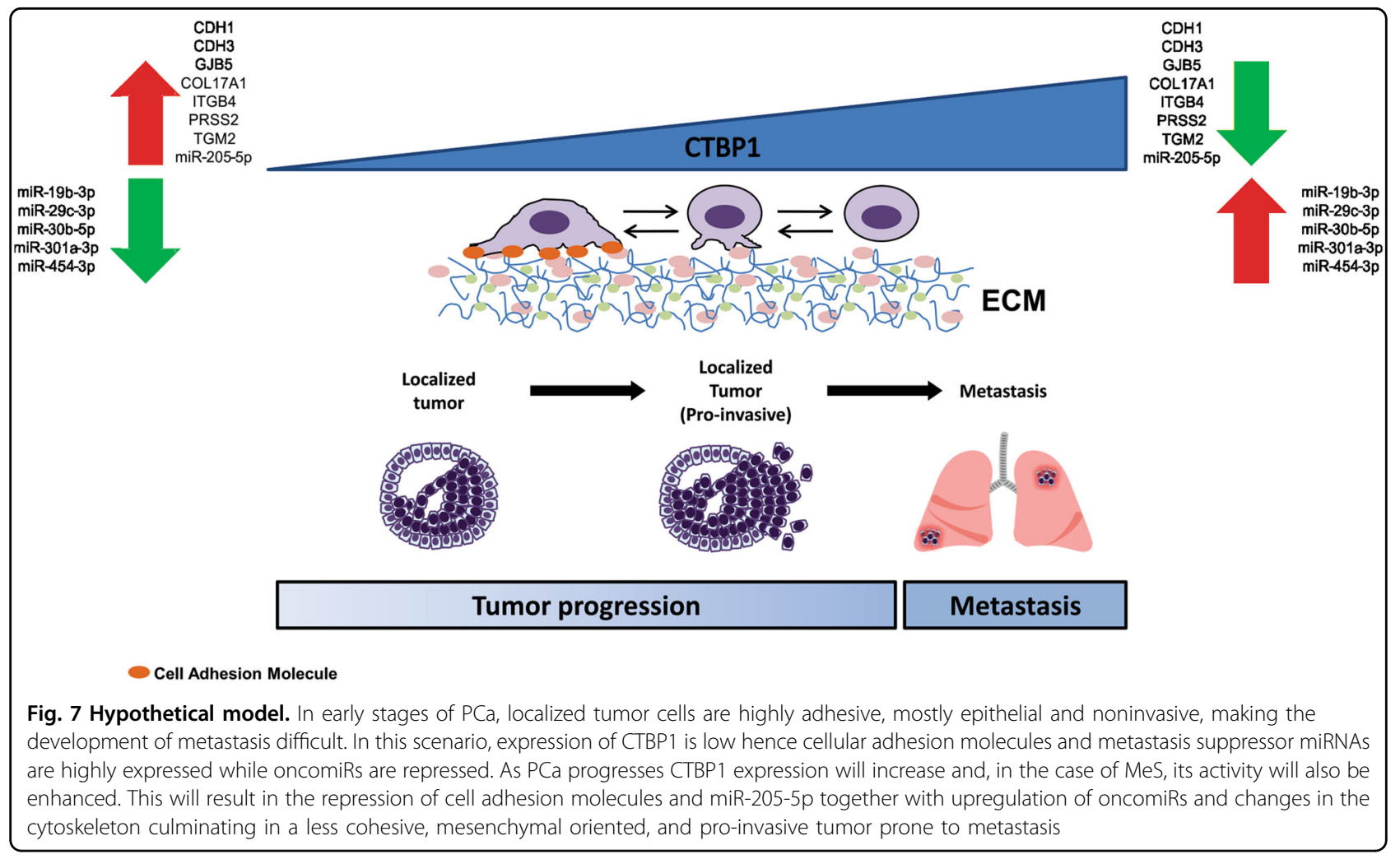

explain the apparent different role for ITGB4 and LCN2 in PCa.

In light of our evidence (see Fig. 7 for hypothetical model) we propose that, as PCa advances in a MeS context, CTBP1 activity and/or expression will increase, resulting in the differential regulation of mRNAs and miRNAs. The repression of cell adhesion molecules and miR-205-5p together with upregulation of oncomiRs and changes in the cytoskeleton will result in a less cohesive, mesenchymal oriented and pro-invasive tumor prone to metastasis. Altogether, our findings explain for the first time why $\mathrm{MeS}$ induces $\mathrm{PCa}$ progression identifying CTBP1 as the crucial player. Further research it's necessary to prove CTBP1 as a candidate for PCa treatment in MeS patients.

\section{Materials and methods}

Cell culture, plasmids, transfections, and treatments

PC3 (ATCC: CRL-1435), 22Rv1 (ATCC: CRL-2505), DU145 (ATCC: HTB-81), LNCaP (ATCC: CRL-1740), and C4- ${ }^{35}$ cell lines and its stable derivatives were grown in RPMI 1640 (Invitrogen) supplemented with 10\% of fetal bovine serum and antibiotics in a $5 \% \mathrm{CO}_{2}$ humidified atmosphere at $37^{\circ} \mathrm{C}$. These cell lines were recently validated at MDA Cancer center (Texas, USA). PC3.shCTBP1 and its control (PC3.PGIPZ) stable cell lines were previously described ${ }^{14}$.
PC3.pcDNA3, pcDNA3.CTBP1 and PC3.ZEB1 cells were generated by transient transfection using $6 \mu \mathrm{g}$ of plasmid and polyethylenimine methodology (PEI-PolySciences Inc.) with PEI:DNA ratio 2:1. CTBP1 plasmid and its control (pcDNA3) were previously described ${ }^{17}$. Zinc Finger E-Box Binding Homeobox 1 (ZEB1) plasmid was previously reported ${ }^{36}$.

\section{RNA isolation, CDNA synthesis, and qPCR (RT-qPCR)}

Total RNA from cells, tumor xenografts, lungs, or plasma was isolated using TriReagent (Molecular Research Center). cDNA synthesis was performed using 2 $\mu \mathrm{g}$ of RNA and RevertAid First Strand kit (Fermentas, Vilnius, Lithuania). Real-time PCR (qPCR) was performed using TAQ Pegasus (Productos Bio-Lógicos, Argentina) in a CFX96 Touch $^{\text {ti }}$ Real-Time PCR Detection System (Bio-Rad). Data were normalized to actin beta $(A C T B)$ or glyceraldehyde-3-phosphate dehydrogenase (GAPDH) and control. Primer sequences are shown in Supplemental Table 1.

\section{miRNA retrotranscription and qPCR (miRNA RT-qPCR)}

miRNAs were retrotranscribed using the stem-loop method as previously described ${ }^{37}$ with some modifications. Briefly, $100 \mathrm{ng}$ of total RNA were preheated at $70^{\circ} \mathrm{C}$ during $5 \mathrm{~min}$ in $14 \mu \mathrm{L}$ containing $0.07 \mu \mathrm{M}$ of stem-loop primer. For plasma samples, cel-39 synthetic miRNA was 
spiked in before RNA extraction, and $4 \mu \mathrm{l}$ of total RNA were used for retrotranscription. Retrotranscription was performed using M-MLV reverse transcriptase (Promega) and incubated in MyGenie96 Thermal Block (Bioneer) for $30 \mathrm{~min}$ at $16^{\circ} \mathrm{C}, 50 \mathrm{~min}$ at $37^{\circ} \mathrm{C}$ and $15 \mathrm{~min}$ at $70^{\circ} \mathrm{C}$. qPCR was performed in $25 \mu \mathrm{L}$ with $0.05-1 \mu \mathrm{L}$ RT product, $1 \mathrm{U}$ Taq DNA polymerase (Pegasus), $4 \mathrm{mM} \mathrm{MgCl}_{2}$, $0.2 \mathrm{mM}$ dNTPs, $3 \times 10^{-5} \mu \mathrm{L}$ Sybrgreen (Sigma), $0.1 \mu \mathrm{M}$ forward primer, and $0.1 \mu \mathrm{M}$ reverse primer. The reactions were incubated in StepOne Plus Real Time PCR (Applied Biosystems) at $94^{\circ} \mathrm{C}$ for $2 \mathrm{~min}$, followed by 40 cycles of $95^{\circ} \mathrm{C}$ for $15 \mathrm{~s}$, annealing temperature for $20 \mathrm{~s}$ and $72^{\circ} \mathrm{C}$ for $25 \mathrm{~s}$. All reactions were run in duplicate. The expression levels of miRNAs were normalized to hsa-miR-103a$3 p$ levels and control. Primer sequences for miRNA RTqPCR are listed in Supplemental Table 1.

\section{Chromatin immunoprecipitation}

ChIP was performed from PC3 cells using specific antibodies for CTBP1 (Santa Cruz Biotechnologies) or nonspecific Gal4 antibody (Santa Cruz Biotechnologies), as previously described ${ }^{14}$. ChIP-DNA was amplified by qPCR using primers located at -0.11 and $0 \mathrm{~kb}$ upstream of the TSS of ITGB4 or $-0.2 \mathrm{~kb}$ upstream of the hemoglobin subunit beta $(H B B)$ TSS (Supplemental Table 1). Fold enrichment was calculated normalizing data to input and Gal4.

\section{Cell adhesion assay}

Cell adhesion assay was performed as previously described with some modifications ${ }^{38}$. Briefly, cells were transfected as indicated and then seeded on a 96-wells culture plate, with or without a collagen coating, followed by 30 or $60 \mathrm{~min}$ of incubation. Afterward culture media was removed and cells were washed twice with phosphate-buffered saline, fixed with methanol and stained with crystal violet (CV) for $10 \mathrm{~min}$. Excess CV was washed with distillated water twice. Stain dissolved in a solution 10\% methanol: $5 \%$ acetic acid was determined with enzyme linked immunosorbent assay Multiskan FC (Thermo Scientific) absorbance at $620 \mathrm{~nm}$.

\section{Immunofluorescence experiments and confocal microscopy}

PC3 cells (transfected or otherwise) were fixed with $8 \%$ paraformaldehyde (20 min, room temperature) and stained with rhodamine-phalloidin ( $1 \mathrm{~h}$, room temperature). Confocal images were acquired by confocal microscopy (FV1000, Olympus, Tokyo, Japan) using an UPlanSApo $60 \times$ oil immersion objective (NA 1/41.35; Olympus), a diode laser of $543 \mathrm{~nm}$ as the excitation source and fluorescence was collected in the range of 555-655 $\mathrm{nm}$. We selected the regions closest to the substrate from which filopodia were clearly defined.
Confocal microscope images were analyzed using ImageJ software (NIH, Bethesda, MD, USA). Cells were considered positive (high-filopodia count) if they presented more than three or more filopodia sets.

\section{MeS and PCa spontaneous metastasis model}

Four-week-old male NOD Scid Gamma (NSG) mice $(N=20)$, were housed under pathogen free conditions following the IBYME's animal care guidelines. Mice were randomized into 2 dietary groups and fed ad libitum during 16 weeks with control diet (CD; $4640 \mathrm{kcal} / \mathrm{kg}, 5 \%$ fat) or HFD $(6,040 \mathrm{kcal} / \mathrm{kg}, 37 \% \mathrm{fat})$. Body weight was monitored once a week. After 12 weeks of diet, mice on each dietary group were randomly distributed into 2 groups and injected s.c with PC3.PGIPZ or PC3. shCTBP1 cells $\left(4.8 \times 10^{6}\right.$ cells per mouse). Tumor volume was determined three times a week and calculated as previously described ${ }^{39}$. Animals were sacrificed in the 16th week and tumor, liver, lung, and blood samples were collected.

\section{Histological and IHC analysis}

Tissue samples collected from $\mathrm{PCa}$ and $\mathrm{MeS}$ mice described above were formalin-fixed and paraffin embedded. For histological analysis, $4 \mu \mathrm{m}$ microscopic sections were stained with hematoxylin-eosin (H\&E) and examined by light microscopy. For IHC analysis of CTBP1, anti-CTBP1 antibody (1:400; BD Biosciences) was used. The procedure was completed using a streptavidin-biotin-complex method (VECTASTAIN ${ }^{\oplus}$ Universal Elite ${ }^{\circledast}$ ABC Kit, Vector Laboratories, Maravai LifeSciences) with 3,3' diaminobenzidine (DAB) as chromogen and examined by light microscopy. IHC evaluation was performed by a pathologist without knowledge of the grouping information.

\section{CTCs assay}

To recover CTCs from peripheral blood, plasma samples (extracted from HFD-fed NSG mice at the time of sacrifice) were treated with ammonium chloride potassium buffer $(1: 1 ; 3 \times 7 \mathrm{~min} ; 1 \times 5 \mathrm{~min})$ in order to lysate erythrocytes and then centrifuge. The cell pellet was resuspended on fresh complete RPMI medium supplemented with puromycin $(1 \mu \mathrm{g} / \mathrm{ml})$ and seeded on a $96-$ wells cell plate. One-week later, cells were photographed with a Q-Color5 Digital Camera (OLYMPUS).

\section{Statistical analysis}

All results are given as mean and standard deviation of three independent experiments unless stated otherwise. Student's $t$ tests or two-way ANOVA followed by Tukey test were performed. Shapiro-Wilk and Levene tests were used to assess normality and homogeneity of variances. ${ }^{*} P<0.05 ;{ }^{* *} P<0.01 ;{ }^{* * *} P<0.001$. 


\section{Acknowledgements}

This research was supported by the Argentinean Agency of Science and Technology (ANPCyT PICT 2014-324; ANPCyT PICT 2015-1345) and by Fundación Williams (Argentina). We thank Dr. Claudia Lanari (Laboratorio de Carcinogénesis Hormonal, IBYME-CONICET, Argentina) for providing the NSG mice.

\section{Author details}

'Laboratorio de Oncología Molecular y Nuevos Blancos Terapéuticos, Instituto de Biología y Medicina Experimental (IBYME), CONICET, Argentina.

${ }^{2}$ Department of Pathology and Cell Biology, Columbia University Medical Center, 630 W. 168th Street, New York, NY 10032, USA. ${ }^{3}$ Departamento de Química Biológica, Universidad de Buenos Aires, Facultad de Ciencias Exactas y Naturales, Laboratorio de inflamación y Cáncer, Buenos Aires, Argentina

\section{Conflict of interest}

The authors declare that they have no conflict of interest.

\section{Publisher's note}

Springer Nature remains neutral with regard to jurisdictional claims in published maps and institutional affiliations.

Supplementary Information accompanies this paper at (https://doi.org/ 10.1038/s41419-019-1535-z).

Received: 7 August 2018 Revised: 25 January 2019 Accepted: 1 March 2019 Published online: 01 April 2019

\section{References}

1. Torre, L. A., Siegel, R. L., Ward, E. M. \& Jemal, A. Global cancer incidence and mortality rates and trends-an update. Cancer Epidemiol. Biomarkers. Prev. 25, 16-27 (2016).

2. Chaffer, C. L. \& Weinberg, R. A. A perspective on cancer cell metastasis. Science 331, 1559-1564 (2011).

3. Sturge, J., Caley, M. P. \& Waxman, J. Bone metastasis in prostate cancer: emerging therapeutic strategies. Nat. Rev. Clin. Oncol. 8, 357-368 (2011).

4. NCEP -ATPIII.. Third Report of the National Cholesterol Education Program (NCEP) expert panel on detection, evaluation, and treatment of high blood cholesterol in adults (adult treatment panel III) final report. Communication 106, 3143-3421 (2002).

5. Grundy, S. M. Metabolic syndrome update. Trends Cardiovasc. Med. 26 364-373 (2016)

6. Nunez, C., Bauman, A., Egger, S., Sitas, F. \& Nair-Shalliker, V. Obesity, physical activity and cancer risks: results from the Cancer, Lifestyle and Evaluation of Risk Study (CLEAR). Cancer Epidemiol. 47, 56-63 (2017).

7. Mandair, D., Rossi, R. E., Pericleous, M., Whyand, T. \& Caplin, M. E. Prostate cancer and the influence of dietary factors and supplements: a systematic review. Nutr. Metab. 11, 30 (2014).

8. Bashir, M. N. Epidemiology of prostate cancer. Asian Pac. J. Cancer Prev. 16, 5137-5141 (2015).

9. Gacci, M. et al. Meta-analysis of metabolic syndrome and prostate cancer Prostate Cancer Prostatic Dis. 1-10. https://doi.org/10.1038/pcan.2017.1 (2017)

10. Peng, Y. \& Croce, C. M. The role of MicroRNAs in human cancer. Signal Transduct. Target Ther. 1, 15004 (2016).

11. Hayes, J., Peruzzi, P. P. \& Lawler, S. MicroRNAs in cancer: biomarkers, functions and therapy. Trends Mol. Med. 20, 460-469 (2014).

12. Massillo, C., Dalton, G. N., Farré, P. L., De Luca, P. \& De Siervi, A. Implications of microRNA dysregulation in the development of prostate cancer. Reproduction 154, R81-R97 (2017)

13. Price, C. \& Chen, J. MicroRNAs in cancer biology and therapy: current status and perspectives. Genes Dis. 1, 53-63 (2014).
14. Moiola, C. P. et al. Prostate tumor growth is impaired by CtBP1 depletion in high-fat diet-fed mice. Clin. Cancer Res. 20, 4086-4095 (2014).

15. Porretti, J. et al. CLCA2 epigenetic regulation by CTBP1, HDACs, ZEB1, EP300 and miR-196b-5p impacts prostate cancer cell adhesion and EMT in metabolic syndrome disease. Int. J. Cancer 143, 897-906 (2018).

16. Blevins, M. A., Huang, M. \& Zhao, R. The role of CtBP1 in oncogenic processes and its potential as a therapeutic target. Mol. Cancer Ther. 16, 981-990 (2017).

17. De Luca, P. et al. CtBP1 associates metabolic syndrome and breast carcinogenesis targeting multiple miRNAs. Oncotarget 7, 18798-18811 (2016).

18. Farré, P. L. et al. CTBP1 and metabolic syndrome induce an mRNA and miRNA expression profile critical for breast cancer progression and metastasis. Oncotarget 9, 13848-13858 (2018).

19. Ichikawa, K. et al. MCRIP1, an ERK substrate, mediates ERK-induced gene silencing during epithelial-mesenchymal transition by regulating the corepressor CtBP. Mol. Cell 58, 35-46 (2015).

20. ZHANG, X.-L. et al. CtBP1 is involved in epithelial-mesenchymal transition and is a potential therapeutic target for hepatocellular carcinoma. Oncol. Rep. $\mathbf{3 0}$ 809-814 (2013).

21. Sahu, S. K. et al. FBXO32 promotes microenvironment underlying epithelialmesenchymal transition via CtBP1 during tumour metastasis and brain development. Nat. Commun. 8, 1523 (2017).

22. Wang, R. et al. Role of transcriptional corepressor CtBP1 in prostate cancer progression. Neoplasia 14, 905-914 (2012)

23. Jacków, J., Löffek, S., Nyström, A., Bruckner-Tuderman, L. \& Franzke, C.-W. Collagen XVII shedding suppresses re-epithelialization by directing keratinocyte migration and dampening mTOR signaling. J. Invest. Dermatol. 136, 1031-1041 (2016).

24. Nishie, W. et al. Context-dependent regulation of collagen XVII ectodomain shedding in skin. Am. J. Pathol. 185, 1361-1371 (2015).

25. Valente, C., Luini, A. \& Corda, D. Components of the CtBP1/BARS-dependent fission machinery. Histochem. Cell Biol. 140, 407-421 (2013).

26. Gregory, P. A. et al. The miR-200 family and miR-205 regulate epithelial to mesenchymal transition by targeting ZEB1 and SIP1. Nat. Cell Biol. 10, 593-601 (2008).

27. Sun $T$ et al. The role of microRNA-221 and microRNA-222 in androgenindependent prostate cancer cell lines. Cancer Res. 69, 3356-3363 (2009).

28. Dhar, S. et al. MTA1-activated Epi-microRNA-22 regulates E-cadherin and prostate cancer invasiveness. FEBS Lett. 591, 924-933 (2017).

29. Gaziel-Sovran, A. et al. miR-30b/30d regulation of GalNAc transferases enhances invasion and immunosuppression during metastasis. Cancer Cell $\mathbf{2 0}$ 104-118 (2011).

30. Tian, S.-B. et al. MiR-30b suppresses tumor migration and invasion by targeting EIF5A2 in gastric cancer. World J. Gastroenterol. 21, 9337 (2015).

31. Banyard, J. et al. Identification of genes regulating migration and invasion using a new model of metastatic prostate cancer. BMC Cancer 14, 387 (2014).

32. Drake, J. M. et al. ZEB1 coordinately regulates laminin-332 and \{beta\}4 integrin expression altering the invasive phenotype of prostate cancer cells. J. Biol. Chem. 285, 33940-33948 (2010).

33. Tung, M.-C. et al. Knockdown of lipocalin-2 suppresses the growth and invasion of prostate cancer cells. Prostate 73, 1281-1290 (2013).

34. Ding, $G$. et al. Over-expression of lipocalin 2 promotes cell migration and invasion through activating ERK signaling to increase SLUG expression in prostate cancer. Prostate 75, 957-968 (2015).

35. Thalmann, G. N. et al. Androgen-independent cancer progression and bone metastasis in the LNCaP model of human prostate cancer. Cancer Res. 54, 2577-2581 (1994)

36. Llorens, M. C. et al. Phosphorylation regulates functions of ZEB1 transcription factor. J. Cell. Physiol. 231, 2205-2217 (2016).

37. Chen, C. et al. Real-time quantification of microRNAs by stem-loop RT-PCR. Nucleic Acids Res. 33, e179 (2005).

38. Fernández, N. B. et al. ROR1 contributes to melanoma cell growth and migration by regulating $\mathrm{N}$-cadherin expression via the PI3K/Akt pathway. Mol. Carcinog. 55, 1772-1785 (2016).

39. De Luca, P. et al. BRCA1 loss induces GADD153-mediated doxorubicin resistance in prostate cancer. Mol. Cancer Res. 9, 1078-1090 (2011). 